\title{
HEIGHT ESTIMATE FOR SPECIAL WEINGARTEN SURFACES OF ELLIPTIC TYPE IN $\mathbb{M}^{2}(c) \times \mathbb{R}$
}

\author{
FILIPPO MORABITO
}

(Communicated by Michael Wolf)

\begin{abstract}
In this article we provide a vertical height estimate for compact special Weingarten surfaces of elliptic type in $\mathbb{M}^{2}(c) \times \mathbb{R}$, i.e. surfaces whose mean curvature $H$ and extrinsic Gauss curvature $K_{e}$ satisfy $H=f\left(H^{2}-K_{e}\right)$ with $4 x\left(f^{\prime}(x)\right)^{2}<1$, for all $x \in[0,+\infty)$. The vertical height estimate generalizes a result by Rosenberg and Sa Earp and applies only to surfaces verifying a height estimate condition. When $c<0$, using also a horizontal height estimate, we show a non-existence result for properly embedded Weingarten surfaces of elliptic type in $\mathbb{H}^{2}(c) \times \mathbb{R}$ with finite topology and one end.
\end{abstract}

\section{INTRODUCTION}

In this work we will consider special Weingarten surfaces of elliptic type in $\mathbb{M}^{2}(c) \times \mathbb{R}$. Here $\mathbb{M}^{2}(c)=\mathbb{S}^{2}(c), \mathbb{R}^{2}$ or $\mathbb{H}^{2}(c)$ depending on the sign of the sectional curvature $c$. If $H$ and $K_{e}$ denote the mean curvature and the extrinsic Gauss curvature of a surface $\Sigma$ respectively, then $\Sigma$ is called a special Weingarten surface if the following identity holds:

$$
H=f\left(H^{2}-K_{e}\right),
$$

with $f \in \mathcal{C}^{0}([0,+\infty))$. Furthermore if $f \in \mathcal{C}^{1}([0,+\infty))$ and $4 x\left(f^{\prime}(x)\right)^{2}<1 \forall x \in$ $[0,+\infty)$, then $f$ is said to be elliptic and $\Sigma$ is said to be a special Weingarten surface of elliptic type, henceforth called a SWET surface.

The study of Weingarten surfaces started with H. Hopf [7, P. Hartman and W. Wintner [8] and S. S. Chern [3, who considered compact Weingarten surfaces in $\mathbb{R}^{3}$. H. Rosenberg and R. Sa Earp [14] showed that compact special Weingarten surfaces in $\mathbb{R}^{3}$ and $\mathbb{H}^{3}$ satisfy an a priori height estimate, assuming also that $f$ satisfies a height estimate condition, and used this fact to prove that the annular ends of a properly embedded special Weingarten surface $M$ are cylindrically bounded. Moreover, if such an $M$ is non-compact and has finite topological type, then $M$ must have more than one end; if $M$ has two ends, then it must be a rotational surface; and if $M$ has three ends, it is contained in a slab. They followed the ideas of Meeks [11] and Korevaar-Kusner-Solomon [10] for non-zero constant mean curvature surfaces in $\mathbb{R}^{3}$. Recently, Aledo-Espinar-Gálvez in 2 obtained a geometric height estimate for SWET surfaces with $f(0) \neq 0$ in $\mathbb{M}^{3}(c), c \leq 0$, with no other hypothesis on $f$.

Received by the editors July 31, 2011 and, in revised form, June 20, 2012; January 29, 2013; March 29, 2013; and April 3, 2013.

2010 Mathematics Subject Classification. Primary 53A10.

Key words and phrases. Special Weingarten surfaces, ellipticity, height estimate. 
R. Sa Earp and E. Toubiana in [16-18] studied rotational special Weingarten surfaces in $\mathbb{R}^{3}$ and $\mathbb{H}^{3}$. In the case $f(0) \neq 0$ (constant mean curvature type), they determined necessary and sufficient conditions for existence and uniqueness of examples whose geometric behaviour is the same as the one of Delaunay surfaces in $\mathbb{R}^{3}$, i.e. unduloids (embedded) and nodoids (non-embedded), which have non-zero constant mean curvature. In the case $f(0)=0$ (minimal type), they established the existence of examples whose geometric behaviour is the same as those of the catenoid of $\mathbb{R}^{3}$, which is the only rotational minimal surface in $\mathbb{R}^{3}$.

By arguments similar to those used by Sa Earp and Toubiana, the author and M. Rodriguez in 12 determined necessary and sufficient conditions for existence and uniqueness of rotational SWET surfaces in $\mathbb{S}^{2} \times \mathbb{R}$ and $\mathbb{H}^{2} \times \mathbb{R}$ of minimal type $(f(0)=0)$.

The reason we focus on SWET surfaces is that the ellipticity of $f$ ensures that the operator obtained by linearization of (1) is elliptic in the sense of Hopf 7 and solutions to (11) satisfy an interior and a boundary maximum principle.

Furthermore we show that an estimate for the height (defined below), similar to one given in [14], holds for SWET surfaces in product manifolds of dimension three under additional assumptions of $f$.

Let $\Sigma$ be a connected orientable hypersurface immersed in $\mathbb{M}^{2}(c) \times \mathbb{R}$. The height function, denoted by $h$, of $\Sigma$ is defined as the restriction to $\Sigma$ of the projection $t: \mathbb{M}^{2}(c) \times \mathbb{R} \rightarrow \mathbb{R}$.

Theorem 1.1 (Height estimate). Let $\Sigma$ be a compact SWET surface embedded in $\mathbb{M}^{2}(c) \times \mathbb{R}$ which is a graph over $\mathbb{M}^{2}(c) \times\{0\}$ with $\partial \Sigma \subset \mathbb{M}^{2}(c) \times\{0\}$. Let $x=H^{2}-K_{e}$. If $f>0, f-2 x f^{\prime}>0$ and $f^{2}+c+x\left(1-4 f f^{\prime}\right)>0$, then

$$
|h| \leqslant 1 / l
$$

where

$$
l=\min _{\Sigma}\left(2 f+\frac{c-K_{e}}{f\left(1-2 f f^{\prime}\right)+2 K_{e} f^{\prime}}\right) .
$$

Corollary 1.2 (Height estimate for cmc surfaces). Let $\Sigma$ be a compact surface having constant mean curvature which is embedded in $\mathbb{M}^{2}(c) \times \mathbb{R}$ and a graph over $\mathbb{M}^{2}(c) \times\{0\}$ such that $\partial \Sigma \subset \mathbb{M}^{2}(c) \times\{0\}$. Suppose that

(1) $c \leq 0, H>\sqrt{\frac{\max _{\Sigma} K_{e}-c}{2}}$, or

(2) $c>0, \max _{\Sigma} K_{e}-c>0, H>\sqrt{\frac{\max _{\Sigma} K_{e}-c}{2}}$, or

(3) $c>0, \max _{\Sigma} K_{e}-c \leq 0, H>0$;

then

$$
|h| \leqslant \frac{H}{2 H^{2}+c-\max _{\Sigma} K_{e}} .
$$

The technique used in 2 to prove a geometric height estimate for SWET surfaces in $\mathbb{M}^{3}(c), c \leq 0$, does not apply to our setting.

Theorem 6.2 in [6] provides a horizontal height estimate for compact surfaces $\Sigma$ with constant curvature or constant mean curvature in $\mathbb{H}^{2}(c) \times \mathbb{R}$ and boundary contained in a vertical plane. The case of surfaces in $\mathbb{S}^{2}(c) \times \mathbb{R}$ is not considered to be $\mathbb{S}^{2}(c)$ compact.

It is possible to prove a similar estimate for SWET surfaces. 
Theorem 1.3 (Horizontal height estimate). Let $P$ denote a vertical plane in $\mathbb{H}^{2}(c) \times \mathbb{R}$. Let $\Sigma$ be a compact $S W E T$ surface in $\mathbb{H}^{2}(c) \times \mathbb{R}$, with $\partial \Sigma \subset P$. Assume that the elliptic function $f \geq H_{0}>\frac{\sqrt{-c}}{2}$. Then for every $p \in \Sigma$, the horizontal distance in $\mathbb{H}^{2}(c) \times \mathbb{R}$ of $p$ to $P$ is bounded by a constant $C$ which does not depend on $\Sigma$.

The proof of Theorem 6.2 in [6] applies verbatim to our setting, with a unique exception: the proof uses the maximum principle to compare $\Sigma$ to a surface $\Sigma_{0}$ that in our case has to be the sphere of constant mean curvature equal to $H_{0}$.

Combining Theorems 1.1 and 1.3 we are in order to prove the following nonexistence result.

Theorem 1.4. There are no properly embedded SWET surfaces in $\mathbb{H}^{2}(c) \times \mathbb{R}$ with finite topology, one end and whose elliptic function $f$ satisfies the hypotheses of Theorems 1.1, 1.3.

Such a theorem generalizes Theorem 7.2 of [6], which applies to surfaces with constant mean curvature $H>1 / 2$ or constant curvature $K>0$ which are properly embedded in $\mathbb{H}^{2} \times \mathbb{R}$.

\section{Proof of Theorem 1.1}

2.1. Preliminaries. Let $\Sigma$ be a oriented connected Riemannian $m$-manifold and let $F: \Sigma \rightarrow \mathbb{M}^{m+1}$ be an isometric immersion of $\Sigma$ into an orientable Riemannian $(m+1)$-manifold $\mathbb{M}^{m+1}$. We choose a normal unit vector field $N$ along $\Sigma$ and define the shape operator $A$ associated with the second fundamental form of $\Sigma$; that is, for any $p \in \Sigma$,

$$
\langle A(X), Y\rangle=-\left\langle\bar{\nabla}_{X} N, Y\right\rangle, X, Y \in T_{p} \Sigma,
$$

where $\bar{\nabla}$ is the Riemannian connection of $\mathbb{M}^{m+1}$.

Let $k_{1}, \ldots, k_{m}$ denote the eigenvalues of $A$. For $1 \leqslant r \leqslant m$, let $S_{r}$ denote the $r$-th symmetric function of $k_{1}, \ldots, k_{m}$ and $T_{r}$ be the $r$-th Newton transformation: $T_{0}=I, T_{r}=S_{r} I-A T_{r-1}$.

If $H_{r}$ denotes the $r$-th mean curvature of $\Sigma$, then $H_{r}=S_{r} / C_{m}^{r}$, where $C_{m}^{r}=$ $\frac{m !}{r !(m-r) !}$.

Let us consider a domain $D \subset \Sigma$ such that its closure $\bar{D}$ is compact with smooth boundary.

Definition 2.1. A variation of $D$ is a differentiable map $\phi:(-\varepsilon, \varepsilon) \times \Sigma \rightarrow \mathbb{M}^{m+1}$, where $\varepsilon>0$, such that for each $s \in(-\varepsilon, \varepsilon)$ the map $\phi_{s}: \Sigma \rightarrow \mathbb{M}^{m+1}$ defined by $\phi_{s}(p)=\phi(s, p)$ is an immersion and $\phi_{0}(p)=F(p)$ for every $p \in \Sigma$ (we recall that $F$ denotes the immersion of $\Sigma$ in $\left.\mathbb{M}^{m+1}\right)$ and $\phi_{s}(p)=F(p)$ for $p \in \Sigma \backslash \bar{D}$ and $s \in(-\varepsilon, \varepsilon)$.

We set

$$
E_{s}(p)=\frac{\partial \phi}{\partial s}(s, p) \quad \text { and } f_{s}=\left\langle E_{s}, N_{s}\right\rangle,
$$

where $N_{s}$ is the unit normal vector field along $\phi_{s}(\Sigma)$. $E$ is called the variational vector field of $\phi$. Let $A_{s}(p)$ be the shape operator of $\phi_{s}(\Sigma)$ at the point $p$ and $S_{r}(s, p)$ the $r$-th symmetric function of the eigenvalues of $A_{s}(p)$.

Definition 2.2. Let $g \in \mathcal{C}^{2}(\Sigma)$. We define $L_{r}(g)=\operatorname{div}\left(T_{r} \nabla g\right), 0 \leqslant r \leqslant m$. 
In [5] M.F. Elbert proved that, for $1 \leqslant r \leqslant m$,

$$
\frac{\partial S_{r}}{\partial s}=L_{r-1}\left(f_{s}\right)+f_{s}\left(S_{1} S_{r}-(r+1) S_{r+1}\right)+f_{s} \operatorname{tr}\left(T_{r-1} \bar{R}_{N}\right)+E_{s}^{T}\left(S_{r}\right),
$$

where $\bar{R}_{N}$ is defined as $\bar{R}_{N}(X)=\bar{R}(N, X) N, \bar{R}$ is the curvature tensor of $\mathbb{M}^{m+1}$ and $E_{s}^{T}$ denotes the tangent part of $E_{s}$.

In the sequel we will consider the case where $\mathbb{M}^{m+1}$ has a special structure: $\mathbb{M}^{m+1}=\mathbb{M}^{m} \times \mathbb{R}$, where $\mathbb{M}^{m}$ is an $m$-dimensional Riemannian manifold.

Definition 2.3. Let $\Sigma$ be a connected orientable hypersurface immersed in $\mathbb{M}^{m} \times \mathbb{R}$. The height function, denoted by $h$, of $\Sigma$ in $\mathbb{M}^{m} \times \mathbb{R}$ is defined as the restriction to $\Sigma$ of the projection $t: \mathbb{M}^{m} \times \mathbb{R} \rightarrow \mathbb{R}$.

The following result has been proved in [4] by X. Cheng and H. Rosenberg.

Lemma 2.4. Let $\Sigma$ be an immersed orientable hypersurface in $\mathbb{M}^{m} \times \mathbb{R}$ (with or without boundary) and $N$ be its normal unit vector field. Then

$$
L_{r}(h)=(r+1) S_{r+1} n,
$$

for $0 \leqslant r \leqslant m$, where $h$ denotes the height function of $\Sigma$, and $n=\left\langle\frac{\partial}{\partial t}, N\right\rangle$.

Lemma 2.5. Let $\Sigma$ be an immersed hypesurface in $\mathbb{M}^{m} \times \mathbb{R}$. Then we have

$$
L_{r}(n)=-n\left(S_{1} S_{r+1}-(r+2) S_{r+2}+\operatorname{tr}\left(T_{r} \bar{R}_{N}\right)\right)-E_{0}^{T}\left(S_{r+1}\right) .
$$

Proof. The proof uses the same argument as the proof of Lemma 4.2 in [4, with the only difference being that in our case $S_{r}$ is not assumed to be constant on $\Sigma$.

Remark 2.6. If either $r=0$ or $r=1$, we get respectively for $m=2$ the following formulae:

$$
\begin{gathered}
L_{0}(n)=\Delta n=-n\left(S_{1}^{2}-2 S_{2}+\operatorname{tr}\left(T_{0} \bar{R}_{N}\right)\right)-E_{0}^{T}\left(S_{1}\right), \\
L_{1}(n)=-n\left(S_{1} S_{2}+\operatorname{tr}\left(T_{1} \bar{R}_{N}\right)\right)-E_{0}^{T}\left(S_{2}\right) .
\end{gathered}
$$

If the manifold $\mathbb{M}^{m}$ has constant sectional curvature, then we are able to express all terms of $L_{r-1}(n)$ given by Lemma 2.5] in terms of the curvatures $S_{r}$.

We denote by $X^{h}$ the horizontal component of $X \in T_{p}\left(\mathbb{M}^{m}(c) \times \mathbb{R}\right)$, by $e_{i}$ the principal directions of $A$ and by $A_{i}$ the restriction of $A$ to the $(m-1)$-dimensional space normal to $e_{i}$.

Lemma 2.7. Let $\Sigma$ denote a hypersurface immersed in $\mathbb{M}^{m}(c) \times \mathbb{R}$. For $0 \leqslant r \leqslant m$, the following holds:

$$
\operatorname{tr}\left(T_{r} \bar{R}_{N}\right)=c(m-r) S_{r}
$$

Proof.

$$
\begin{aligned}
& \operatorname{tr}\left(T_{r} \bar{R}_{N}\right)(p)=\sum_{i}\left\langle e_{i}, T_{r+1} \bar{R}_{N}\left(e_{i}\right)\right\rangle(p)=\sum_{i} S_{r+1}\left(A_{i}\right) \bar{R}\left(N, e_{i}, N, e_{i}\right)(p) \\
& =\sum_{i} S_{r+1}\left(A_{i}\right) K\left(e_{i}^{h}, N^{h}\right)\left|e_{i}^{h} \wedge N^{h}\right|^{2}(p)=c \sum_{i} S_{r+1}\left(A_{i}\right)=c(m-r) S_{r} .
\end{aligned}
$$

Remark 2.8. If either $r=0$ or $r=1$, we get respectively for $m=2$ the following formulae:

$$
\begin{gathered}
\operatorname{tr}\left(T_{0} \bar{R}_{N}\right)=2 c S_{0}=2 c, \\
\operatorname{tr}\left(T_{1} \bar{R}_{N}\right)=c S_{1}=2 c H_{1} .
\end{gathered}
$$


In the next section we will use the results presented here to show that the special Weingarten surfaces of elliptic type satisfy an interior and a boundary maximum principle and a height estimate under additional conditions.

2.2. Maximum principle for special Weingarten surfaces. Let $\Sigma$ be an oriented connected hypersurface immersed in $\mathbb{M}^{m}(c) \times \mathbb{R}$ and $f \in \mathcal{C}^{1}([0, \infty))$. Let us suppose that the first and second mean curvatures $H_{1}(s), H_{2}(s)$ of $\phi_{s}(\Sigma)$ (see Definition 2.1) satisfy

$$
H_{1}-f\left(H_{1}^{2}-H_{2}\right)=0 .
$$

The first variation of the left member of this identity at $s=0$ gives us

$$
\left(\left(1-2 H_{1} f^{\prime}\left(H_{1}^{2}-H_{2}\right)\right) \frac{\partial H_{1}}{\partial s}+f^{\prime}\left(H_{1}^{2}-H_{2}\right) \frac{\partial H_{2}}{\partial s}\right)_{\left.\right|_{\{s=0\}}}=0 .
$$

From (3), the principal parts of $\partial_{s} H_{1}(0)=\frac{1}{m} \partial_{s} S_{1}(0)$ and $\partial_{s} H_{2}(0)=\frac{2}{m(m-1)} \partial_{s} S_{2}(0)$ are respectively $L_{0} / m$ and $\frac{2}{m(m-1)} L_{1}$.

When $m=2$ the linearized operator of (41) reduces to

$$
L_{f}=\left(\frac{1-2 f f^{\prime}}{2}\right) \Delta+f^{\prime} L_{1} .
$$

As in [14, page 294], we can prove the following lemma.

Lemma 2.9. If the function $f$ is elliptic, that is, $4 x\left(f^{\prime}(x)\right)^{2}<1$ for all $x \geq 0$, then the eigenvalues of the operator $L_{f}$ are positive. In other terms $L_{f}$ is elliptic.

Remark 2.10. Lemma 2.9 says that $H_{1}=f\left(H_{1}^{2}-H_{2}\right)$ is elliptic in the sense of Hopf and the solutions of this equation satisfy an interior and a boundary maximum principle (see [7, pages 156-158]).

Let $\Sigma_{1}, \Sigma_{2}$ be two oriented special Weingarten surfaces in $\mathbb{M}^{2}(c) \times \mathbb{R}$ satisfying (11) for the same function $f$, whose unit normal vectors coincide at a common point $p$. For $i=1,2$, we can write $\Sigma_{i}$ locally around $p$ as a graph of a function $u_{i}$ over a domain in $T_{p} \Sigma_{1}=T_{p} \Sigma_{2}$ (in exponential coordinates). We will say that $\Sigma_{1}$ is above $\Sigma_{2}$ in a neighbourhood of $p$, and we will write $\Sigma_{1} \geq \Sigma_{2}$ if $u_{1} \geq u_{2}$.

Proposition 2.11 (Maximum Principle [7]). Let $\Sigma_{1}, \Sigma_{2}$ be two special Weingarten surfaces in $\mathbb{M}^{2}(c) \times \mathbb{R}$ with respect to the same elliptic function $f$. Let us suppose that

- $\Sigma_{1}$ and $\Sigma_{2}$ are tangent at an interior point $p \in \Sigma_{1} \cap \Sigma_{2}$ or

- there exists $p \in \partial \Sigma_{1} \cap \partial \Sigma_{2}$ such that both $T_{p} \Sigma_{1}=T_{p} \Sigma_{2}$ and $T_{p} \partial \Sigma_{1}=T_{p} \partial \Sigma_{2}$.

Also suppose that the unit normal vectors of $\Sigma_{1}, \Sigma_{2}$ coincide at $p$. If $\Sigma_{1} \geq \Sigma_{2}$ in a neighbourhood $U$ of $p$, then $\Sigma_{1}=\Sigma_{2}$ in $U$. In the case $\Sigma_{1}, \Sigma_{2}$ have no boundary, $\Sigma_{1}=\Sigma_{2}$.

To show the main theorem we need the following result.

Lemma 2.12. If $H_{1}(s)$ and $H_{2}(s)$ verify $H_{1}-f\left(H_{1}^{2}-H_{2}\right)=0$ for each $s$, then

$$
\left(1-2 f f^{\prime}\right) E_{s}^{T}\left(H_{1}\right)+f^{\prime} E_{s}^{T}\left(H_{2}\right)=0 .
$$


Proof. It is sufficient to find the explicit expression of $E_{s}^{T}\left(H_{1}-f\left(H_{1}^{2}-H_{2}\right)\right)$ and to use the fact that it vanishes. It holds that

$$
E_{s}^{T}\left(H_{1}\right)-E_{s}^{T} f\left(H_{1}^{2}-H_{2}\right)=E_{s}^{T}\left(H_{1}\right)-2 f f^{\prime} E_{s}^{T}\left(H_{1}\right)+f^{\prime} E_{s}^{T}\left(H_{2}\right)=0 .
$$

That is, $\left(1-2 f f^{\prime}\right) E_{s}^{T}\left(H_{1}\right)+f^{\prime} E_{s}^{T}\left(H_{2}\right)=0$.

Now we give the proof of Theorem 1.1 .

Proof. Let $m$ denote the maximum of $h$ on $\Sigma$. It is sufficient to give the proof assuming that $\Sigma$ is a graph on the slice $\{t=0\}$, where $t$ denotes the coordinate on $\mathbb{R}$. Indeed by coming from infinity with horizontal slices and applying the Alexandrov reflection to $\Sigma$, we see that the part of $\Sigma$ above the plane $t=m / 2$ is a graph over a domain in this plane. The estimate we are going to prove is $|h| \leqslant 1 / l$ when $\Sigma$ is a graph. We can assume $h \geqslant 0$ on $\Sigma$; otherwise we apply the following argument to the part of $\Sigma$ above $\{t=0\}$ and to the part of $\Sigma$ below $\{t=0\}$. We orient the unit normal vector to $\Sigma$ so that $n \leqslant 0$. We set $\varphi=l h+n$. On $\partial \Sigma$ we have $\varphi=n \leqslant 0$. If we show that $L_{f} \varphi \geqslant 0$ on $\Sigma$, from ellipticity of $L_{f}$ and the maximum principle we get that $\varphi \leqslant 0$ on $\Sigma$, that is, $h \leqslant-n / l \leqslant 1 / l$. By Lemma 2.4, Remarks 2.6] and 2.8, and Lemma 2.12 we get

$$
\begin{gathered}
L_{f}(\varphi)=L_{f}(l h+n)=l L_{f}(h)+L_{f}(n) \\
=l\left(\left(\frac{1-2 f f^{\prime}}{2}\right) \Delta h+f^{\prime} L_{1} h\right)+\left(\frac{1-2 f f^{\prime}}{2}\right) \Delta n+f^{\prime} L_{1} n \\
=l\left(\left(\frac{1-2 f f^{\prime}}{2}\right) 2 H_{1} n+2 f^{\prime} H_{2} n\right) \\
-\left(\frac{1-2 f f^{\prime}}{2}\right)\left(\left(4 H_{1}^{2}-2 H_{2}+2 c\right) n+2 E_{0}^{T}\left(H_{1}\right)\right)-f^{\prime}\left(\left(2 H_{1} H_{2}+2 c H_{1}\right) n+E_{0}^{T}\left(H_{2}\right)\right) \\
=-n\left(\left(1-2 f f^{\prime}\right)\left(2 H_{1}^{2}-H_{2}+c\right)+f^{\prime}\left(2 H_{1} H_{2}+2 c H_{1}\right)\right) \\
+l\left(\left(1-2 f f^{\prime}\right) H_{1} n+2 f^{\prime} H_{2} n\right)-\left(1-2 f f^{\prime}\right) E_{0}^{T}\left(H_{1}\right)+f^{\prime} E_{0}^{T}\left(H_{2}\right) \\
=-n\left(\left(1-2 f f^{\prime}\right)\left(2 H_{1}^{2}-H_{2}+c-l H_{1}\right)+f^{\prime}\left(2 H_{1} H_{2}+2 c H_{1}-2 l H_{2}\right)\right) .
\end{gathered}
$$

We replace $H_{1}$ by $f$ and $H_{1}^{2}-H_{2}$ by $x$. We get

$$
\begin{gathered}
-n\left(\left(1-2 f f^{\prime}\right)\left(f^{2}+x+c-l f\right)+f^{\prime}\left(2 f\left(f^{2}-x\right)+2 c f-2 l\left(f^{2}-x\right)\right)\right) \\
=-n\left(f^{2}-l f+x+2 x l f^{\prime}-4 x f f^{\prime}+c\right) .
\end{gathered}
$$

Such a quantity can be written as

$$
-n\left(f(f-l)\left(1-4 x f^{\prime 2}\right)+c+x\left(1-2 f f^{\prime}\right)^{2}+2 x l f^{\prime}\left(1-2 f f^{\prime}\right)\right),
$$

which is non-negative if

$$
\begin{gathered}
f(f-l)\left(1-4 x f^{\prime 2}\right)+c+x\left(1-2 f f^{\prime}\right)^{2}+2 x l f^{\prime}\left(1-2 f f^{\prime}\right) \\
=f^{2}+c+x\left(1-4 f f^{\prime}\right)-l\left[f\left(1-4 x f^{\prime 2}\right)-2 x f^{\prime}\left(1-2 f f^{\prime}\right)\right] \\
=f^{2}+c+x\left(1-4 f f^{\prime}\right)-l\left[f-2 x f^{\prime}\right] \geqslant 0 .
\end{gathered}
$$

Suppose that

$$
f-2 x f^{\prime}>0
$$

Then

$$
l \leqslant \frac{f^{2}+c+x\left(1-4 f f^{\prime}\right)}{f-2 x f^{\prime}}=f+\frac{c+x\left(1-2 f f^{\prime}\right)}{f-2 x f^{\prime}} .
$$


As $x=f^{2}-K_{e}$ we have

$$
l \leqslant f+\frac{c-K_{e}+f^{2}-2\left(f^{2}-K_{e}\right) f f^{\prime}}{f-2\left(f^{2}-K_{e}\right) f^{\prime}}=2 f+\frac{c-K_{e}}{f-2\left(f^{2}-K_{e}\right) f^{\prime}}=: L .
$$

If we assume $f^{2}+c+x\left(1-4 f f^{\prime}\right)>0$, then $L>0$. We conclude that the best constant $l$ is

$$
l=\min _{\Sigma}\left(2 f+\frac{c-K_{e}}{f-2\left(f^{2}-K_{e}\right) f^{\prime}}\right)=\min _{\Sigma}\left(2 f+\frac{c-K_{e}}{f\left(1-2 f f^{\prime}\right)+2 K_{e} f^{\prime}}\right) .
$$

Now we give the proof of Corollary 1.2 .

Proof. If $f^{\prime}=0$, that is, $\Sigma$ has constant mean curvature, we get the estimate

$$
h \leqslant \frac{1}{l}=\max _{\Sigma} \frac{H}{2 H^{2}+c-K_{e}}=\frac{H}{2 H^{2}+c-\max _{\Sigma} K_{e}}
$$

under the assumption $2 H^{2}+c-\max _{\Sigma} K_{e}>0$.

Remark 2.13. If $c<0$, then the estimate of Corollary 1.2 holds if $H>\sqrt{\frac{\max _{\Sigma} K_{e}-c}{2}}$. We observe that $\sqrt{\frac{\max _{\Sigma} K_{e}-c}{2}} \geqslant \sqrt{\frac{-c}{2}}$. As a consequence such an estimate is not sharp because the smallest value of the mean curvature of a compact surface in $\mathbb{H}^{2}(c) \times \mathbb{R}$ equals $\sqrt{-c} / 2$. Optimal estimates have been derived in 1 . If $c=0$ and $\Sigma$ is a semisphere (in particular $H^{2}=K_{e}$ holds), we get the well known estimate $|h| \leqslant 1 / H$.

\subsection{Proof of Theorem 1.4.}

Proof. Let us suppose by absurdity that $S$ is a properly embedded SWET surface with finite topology and one end with respect to a function $f$ which also satisfies the hypotheses of Theorems 1.1, 1.3.

Let us denote by $D$ a constant bigger than $\max \{C, d\}$, where $C$ is the bound for the horizontal height given by Theorem 1.3 and $d$ is the horizontal diameter of the sphere of constant mean curvature equal to $H_{0}$.

Let $p$ denote a point in $S$ and $\gamma$ a horizontal geodesic containing $p$. Let $p_{1}, p_{2}$ be two points in $\gamma$ such that $\operatorname{dist}_{\mathbb{H}^{2}(c)}\left(p, p_{1}\right)=\operatorname{dist}_{\mathbb{H}^{2}(c)}\left(p_{1}, p_{2}\right)=D, \operatorname{dist}_{\mathbb{H}^{2}(c)}\left(p, p_{2}\right)=$ $2 D$. Let $P_{1}, P_{2}$ be two vertical totally geodesic planes intersecting orthogonally with $\gamma$ at $p_{1}, p_{2}$ respectively.

We now use the following variant of the Plane Separation Lemma proved in 13 . Its proof is exactly the same.

Lemma 2.14. Let $S$ be a properly embedded $S W E T$ annulus in $\mathbb{H}^{2}(c) \times \mathbb{R}$. Suppose that $f \geq H_{0}>\frac{\sqrt{-c}}{2}$. Let $P_{1}$ and $P_{2}$ be two vertical totally geodesic planes. Assume that the distance between $P_{1}$ and $P_{2}$ is bigger than the horizontal diameter of the sphere of constant mean curvature $H_{0}$. Denote by $P_{1}^{+}$and $P_{2}^{+}$the components of $\mathbb{H}^{2}(c) \times \mathbb{R} \backslash P_{j}$ such that $P_{1}^{+} \cap P_{2}^{+}=\emptyset$. Then all the connected components of $S \cap P_{1}^{+}$ or $S \cap P_{2}^{+}$are compact.

The distance between $P_{1}$ and $P_{2}$ equals $D>d$; then the previous lemma applies. Suppose that all of the connected components of $S \cap P_{1}^{+}$are compact. By construction the plane $P_{1}$ is at distance $D$ to the point $p \in S \cap P_{1}^{+}$. As $D>C$, $C$ being the bound for the horizontal distance, this would contradict Theorem 1.3 . 
Then all the connected components of $S \cap P_{2}^{+}$are compact. By Theorem [1.3 the points of $S \cap P_{2}^{+}$are at a distance to $P_{2}$ which has to be smaller than $C$.

If we use the same argument after replacing $\gamma$ by every other horizontal geodesic line passing by $p$, we can prove that $S$ is located at finite distance to $\{p\} \times \mathbb{R}$. In other words, $S$ is contained in a vertical cylinder.

As by hypothesis $S$ has exactly one end, we can assume that $S$ is contained in the halfspace $\{t \leq 0\}$ and is tangent to $\{t=0\}$.

For $z<0$ we consider the reflection in $\{t=z\}$ of the compact piece $S_{z}$ of $S$ contained in $\{z \leq t \leq 0\}$. We will show that $S_{z}$ is a vertical graph on $\{t=z\}$ for any $z<0$. We denote by $S_{z}^{R}$ the reflection of $S_{z}$ in $\{t=z\}$. We observe that $S_{z}^{R}$ does not have common tangent points with $S$. Otherwise by the Maximum Principle, Proposition 2.11, we could conclude that $S$ is compact. For the same reason $S_{z}^{R}$ and $S_{z}$ are not orthogonal to $\{t=z\}$ for any $z<0$. This proves that $S_{z}$ is a graph.

Now we can choose $z_{0}<0$ with $\left|z_{0}\right|$ big enough so that the height of the compact graph $S_{z_{0}}$ (having boundary on the plane $\left\{t=z_{0}\right\}$ ) is arbitrarily big. This contradicts Theorem 1.1.

\section{ACKNOWLEDGEMENT}

The author wishes to express his gratitude to the referee for valuable suggestions.

\section{References}

[1] Juan A. Aledo, José M. Espinar, and José A. Gálvez, Height estimates for surfaces with positive constant mean curvature in $\mathbb{M}^{2} \times \mathbb{R}$, Illinois J. Math. 52 (2008), no. 1, 203-211. MR.2507241 (2010e:53006)

[2] Juan A. Aledo, José M. Espinar, and José A. Gálvez, The Codazzi equation for surfaces, Adv. Math. 224 (2010), no. 6, 2511-2530, DOI 10.1016/j.aim.2010.02.007. MR2652214 (2011j:53100)

[3] Shiing-shen Chern, On special W-surfaces, Proc. Amer. Math. Soc. 6 (1955), 783-786. MR0074857 (17,657h)

[4] Xu Cheng and Harold Rosenberg, Embedded positive constant $r$-mean curvature hypersurfaces in $M^{m} \times \mathbf{R}$, An. Acad. Brasil. Ciênc. 77 (2005), no. 2, 183-199 (English, with English and Portuguese summaries), DOI 10.1590/S0001-37652005000200001. MR2137392 (2006e:53105)

[5] Maria Fernanda Elbert, Constant positive 2-mean curvature hypersurfaces, Illinois J. Math. 46 (2002), no. 1, 247-267. MR1936088 (2003g:53103)

[6] José M. Espinar, José A. Gálvez, and Harold Rosenberg, Complete surfaces with positive extrinsic curvature in product spaces, Comment. Math. Helv. 84 (2009), no. 2, 351-386, DOI 10.4171/CMH/165. MR.2495798 (2010c:53086)

[7] Heinz Hopf, Differential geometry in the large, Notes taken by Peter Lax and John Gray, with a preface by S. S. Chern, Lecture Notes in Mathematics, vol. 1000, Springer-Verlag, Berlin, 1983. MR707850 (85b:53001)

[8] Philip Hartman and Aurel Wintner, Umbilical points and W-surfaces, Amer. J. Math. 76 (1954), 502-508. MR0063082 (16,68a)

[9] Nicholas J. Korevaar, Rob Kusner, William H. Meeks III, and Bruce Solomon, Constant mean curvature surfaces in hyperbolic space, Amer. J. Math. 114 (1992), no. 1, 1-43, DOI 10.2307/2374738. MR:1147718 (92k:53116)

[10] Nicholas J. Korevaar, Rob Kusner, and Bruce Solomon, The structure of complete embedded surfaces with constant mean curvature, J. Differential Geom. 30 (1989), no. 2, 465-503. MR.1010168 (90g:53011)

[11] William H. Meeks III, The topology and geometry of embedded surfaces of constant mean curvature, J. Differential Geom. 27 (1988), no. 3, 539-552. MR940118 (89h:53025) 
[12] Filippo Morabito and M. Magdalena Rodríguez, Classification of rotational special Weingarten surfaces of minimal type in $\mathbb{S}^{2} \times \mathbb{R}$ and $\mathbb{H}^{2} \times \mathbb{R}$, Math. Z. 273 (2013), no. 1-2, 379-399, DOI 10.1007/s00209-012-1010-3. MR3010166

[13] Barbara Nelli and Harold Rosenberg, Simply connected constant mean curvature surfaces in $\mathbb{H}^{2} \times \mathbb{R}$, Michigan Math. J. 54 (2006), no. 3, 537-543, DOI 10.1307/mmj/1163789914. MR2280494 (2008f:53007)

[14] Harold Rosenberg and Ricardo Sa Earp, The geometry of properly embedded special surfaces in $\mathbf{R}^{3}$, e.g., surfaces satisfying $a H+b K=1$, where $a$ and $b$ are positive, Duke Math. J. 73 (1994), no. 2, 291-306, DOI 10.1215/S0012-7094-94-07314-6. MR1262209 (95b:53010)

[15] Richard M. Schoen, Uniqueness, symmetry, and embeddedness of minimal surfaces, J. Differential Geom. 18 (1983), no. 4, 791-809 (1984). MR730928 (85f:53011)

[16] Ricardo Sa Earp and Eric Toubiana, Sur les surfaces de Weingarten spéciales de type minimal, Bol. Soc. Brasil. Mat. (N.S.) 26 (1995), no. 2, 129-148 (French, with English summary), DOI 10.1007/BF01236989. MR1364263 (96j:53006)

[17] Ricardo Sa Earp and Eric Toubiana, Classification des surfaces de type Delaunay, Amer. J. Math. 121 (1999), no. 3, 671-700 (French, with English summary). MR1738404 (2001b:53004)

[18] Ricardo Sa Earp and Eric Toubiana, Symmetry of properly embedded special Weingarten surfaces in $\mathbf{H}^{3}$, Trans. Amer. Math. Soc. 351 (1999), no. 12, 4693-4711, DOI 10.1090/S00029947-99-02511-8. MR:1675186(2000c:53007)

Korea Institute for Advanced Study, Cheongnyangni 2-dong, Dongdaemun-gu, Seoul, 130-722, South Korea

Current address: Department of Mathematical Sciences, Korea Advanced Institute Science Technology, 291 Daehak-ro, Yuseong-gu, Daejeon, 305-701, South Korea 